\title{
Positive solutions of a nonlinear algebraic system with sign-changing coefficient matrix
}

Yanping Jia', Ying Gao' ${ }^{1}$ Wenying Feng ${ }^{2}$ and Guang Zhang ${ }^{3 *}$

\section{"Correspondence:}

lxyzhg@tjcu.edu.cn

${ }^{3}$ School of Science, Tianjin

University of Commerce, Tianjin

300134, P.R. China

Full list of author information is

available at the end of the article

\section{Springer}

\begin{abstract}
Existence of positive solutions for the nonlinear algebraic system $x=\lambda G F(x)$ has been extensively studied when the $n \times n$ coefficient matrix $G$ is positive or nonnegative. However, to the best of our knowledge, few results have been obtained when the coefficient matrix changes sign. In this case, some commonly applied analysis methods such as the cone theory, the Krein-Rutman theorem, the monotone iterative techniques, and so on cannot be directly applied. In this note, we prove the existence of positive solutions for the above nonlinear algebraic system with sign-changing coefficient matrix taking the advantages of the classical Brouwer fixed point theorem combined with a decomposition condition on the coefficient matrix. We provide an example in solving a second-order difference equation with periodic boundary conditions to illustrate the applications of the results.
\end{abstract}

MSC: 39A10

Keywords: Fixed point theorem; Nonlinear algebraic system; Positive solution; Difference equation

\section{Introduction}

The nonlinear algebraic system

$$
x=\lambda G F(x)
$$

has interesting applications in boundary value problems, complex systems, networks, optimization, and many other areas [7-9, 12, 14, 25, 27], where $\lambda \in \mathbb{R}$ is a parameter, $x=\operatorname{col}\left(x_{1}, x_{2}, \ldots, x_{n}\right)$,

$$
F(x)=\operatorname{col}\left(f\left(x_{1}\right), f\left(x_{2}\right), \ldots, f\left(x_{n}\right)\right),
$$

$f: \mathbb{R} \rightarrow \mathbb{R}$ is continuous, and $G=\left(g_{i j}\right)_{n \times n}$ is an $n \times n$ square matrix. The existence of positive solutions for system (1) has been extensively studied in the literature; see $[2,3,5,6,10,11,13,15,17,20,21,24,27-29]$ and the references therein. However, to the best of our knowledge, almost all obtained results require that the coefficient matrix $G \geq 0$ or $G>0$, where $G \geq 0$ if $g_{i j} \geq 0$ and $G>0$ if $g_{i j}>0$ for $(i, j) \in[1, n] \times[1, n]$, and $[1, n]=\{1,2, \ldots, n\}$.

(c) The Author(s) 2020. This article is licensed under a Creative Commons Attribution 4.0 International License, which permits use, sharing, adaptation, distribution and reproduction in any medium or format, as long as you give appropriate credit to the original author(s) and the source, provide a link to the Creative Commons licence, and indicate if changes were made. The images or other third party material in this article are included in the article's Creative Commons licence, unless indicated otherwise in a credit line to the material. If material is not included in the article's Creative Commons licence and your intended use is not permitted by statutory regulation or exceeds the permitted use, you will need to obtain permission directly from the copyright holder. To view a copy of this licence, visit http://creativecommons.org/licenses/by/4.0/. 
An $n \times n$ square matrix $G$ is called a sign-changing coefficient matrix if its elements change the sign. When the coefficient matrix $G$ changes the sign, it is difficult to construct a suitable positive order cone to apply the topological degree theory for fixed points or monotone approximation sequences. Therefore some commonly applied analysis methods such as the cone theory, the Krein-Rutman theorem, the monotone iterative techniques, and so on cannot be applied. Thus we need to seek new approaches. In this note, we take the advantages of the classical Brouwer fixed point theorem $[4,26]$ combined with a decomposition condition for the coefficient matrix. We prove the existence of a positive solution for system (1) when the parameter $\lambda$ is in an interval $\left(0, \lambda_{0}\right)$ with $\lambda_{0}>0$. We give a particular example to show an easy calculation of $\lambda_{0}$. We apply the results to prove the solvability of a second-order periodic boundary value problem. It is also possible to further extend the idea to operator equations in general Banach spaces.

The present work is motivated by Ma [19]. In fact, there are many publications considering the existence of solutions for differential equations with sign-changing coefficients; for example, see $[16,18,19,22,23]$ and the references therein. It is well known that the Green functions are structured when these problems are considered. The corresponding discrete case for this type of problems can be formed as system (1) with coefficient matrix $G$ containing columns with all negative values. However, this method cannot be applied to system (1) since the negative elements of $G$ are not required to be in specified positions. In other words, system (1) does not represent a discrete analogue of the corresponding differential equation in most cases; see [1]. Moreover, the simplicity of the discrete setting enables us to successfully apply some computational tools that do not seem to have continuous counterparts.

\section{Main results}

For convenience, we first give the Brouwer fixed point theorem, which is the foundation of many fixed point theorems $[4,26]$.

Lemma 1 (Brouwer Fixed Point Theorem (1912)) Let $M$ be a nonempty convex compact subset of $\mathbb{R}^{n}$, and let $F: M \rightarrow M$ be a continuous mapping. Then $F$ has a fixed point.

From Lemma 1 we can obtain the following result that will be valuable for the proof of Theorem 1.

Lemma 2 Suppose that $\bar{f}: \mathbb{R} \rightarrow \mathbb{R}$ is continuous and $|\bar{f}(u)| \leq N$ for some constant $N>0$. Then for every $\lambda \in \mathbb{R}$, the problem

$$
x=\lambda G \bar{F}(x)
$$

has a solution $x^{\lambda}$, where $\bar{F}(x)=\operatorname{col}\left(\bar{f}\left(x_{1}\right), \bar{f}\left(x_{2}\right), \ldots, \bar{f}\left(x_{n}\right)\right)$.

Proof For any $x \in \mathbb{R}^{n}$, we have

$$
\|\lambda G \bar{F}(x)\|_{\infty} \leq n|\lambda| \max _{i, j \in[1, n]}\left|g_{i j}\right| N \triangleq \delta
$$

where $\|x\|_{\infty}=\max _{i \in[1, n]}\left\{\left|x_{i}\right|\right\}$. The Brouwer fixed point theorem ensures that system (2) has a solution $x^{\lambda} \in M=[-\delta, \delta]^{n}$. The proof is complete. 
Theorem 1 Let $f: \mathbb{R} \rightarrow \mathbb{R}$ be a continuous function with $f(0)>0$, and let

$$
\sum_{j=1}^{n} g_{i j}>0 \quad \text { for } i \in[1, n]
$$

Then there exists $\lambda_{0}>0$ such that problem (1) has a positive solution for any $\lambda \in\left(0, \lambda_{0}\right)$.

Proof Since $f$ is continuous, we can choose a fixed $M>0$ such that $f(u)>0$ for $u \in[0, M]$. Define $\bar{f}(u)$ as follows:

$$
\bar{f}(u)= \begin{cases}f(0), & u \leq 0 \\ f(u), & 0<u \leq M, \\ f(M), & u>M\end{cases}
$$

Lemma 2 implies that system (2) has a solution $x^{\lambda}$.

Denote

$$
g_{i j}^{+}=\max \left\{g_{i j}, 0\right\} \quad \text { and } \quad g_{i j}^{-}=\max \left\{-g_{i j}, 0\right\} \quad \text { for } i, j \in[1, n] \text {. }
$$

Let

$$
g_{i j}=g_{i j}^{+}-g_{i j}^{-} \quad \text { for } i, j \in[1, n] .
$$

From (3) we have

$$
\sum_{j=1}^{n} g_{i j}^{+}>\sum_{j=1}^{n} g_{i j}^{-} \quad \text { for } i \in[1, n] .
$$

There exists $\varepsilon>0$ such that

$$
\sum_{j=1}^{n} g_{i j}^{+}-(1+\varepsilon) \sum_{j=1}^{n} g_{i j}^{-}>0 \quad \text { for } i \in[1, n] .
$$

For fixed $\gamma \in(0, \varepsilon /(2+\varepsilon))$, the continuity of $\bar{f}$ implies that there exists $\delta \in(0, M)$ such that

$$
(1-\gamma) \bar{f}(0)<\bar{f}(u)<(1+\gamma) \bar{f}(0) \quad \text { for }|u|<\delta .
$$

Since

$$
x_{i}^{\lambda}=\lambda \sum_{j=1}^{n} g_{i j} \bar{f}\left(x_{j}^{\lambda}\right) \quad \text { for } i \in[1, n],
$$

it follows that there exists $\lambda_{0}>0$ such that $\left\|x^{\lambda}\right\|_{\infty}<\delta$ for $\lambda \in\left(0, \lambda_{0}\right)$ and that

$$
x_{i}^{\lambda}=\lambda \sum_{j=1}^{n} g_{i j} \bar{f}\left(x_{j}^{\lambda}\right)
$$




$$
\begin{aligned}
= & \lambda\left\{\sum_{j=1}^{n} g_{i j}^{+} \bar{f}\left(x_{j}^{\lambda}\right)-\sum_{j=1}^{n} g_{i j}^{-} \bar{f}\left(x_{j}^{\lambda}\right)\right\} \\
> & \lambda\left\{(1-\gamma) \bar{f}(0) \sum_{j=1}^{n} g_{i j}^{+}-(1+\gamma) \bar{f}(0) \sum_{j=1}^{n} g_{i j}^{-}\right\} \\
= & \lambda(1-\gamma) \bar{f}(0)\left\{\sum_{j=1}^{n} g_{i j}^{+}-\frac{1+\gamma}{1-\gamma} \sum_{j=1}^{n} g_{i j}^{-}\right\} \\
= & \lambda(1-\gamma) \bar{f}(0)\left\{\sum_{j=1}^{n} g_{i j}^{+}-(1+\varepsilon) \sum_{j=1}^{n} g_{i j}^{-}\right\} \\
& +\lambda(1-\gamma) \bar{f}(0)\left\{1+\varepsilon-\frac{1+\gamma}{1-\gamma}\right\} \sum_{j=1}^{n} g_{i j}^{-} \\
> & 0 .
\end{aligned}
$$

The proof is complete.

Remark 1 The proof of Theorem 1 is motivated by Ma [19]. However, condition (3) is new and weaker than Theorem 2.2 in [19].

Remark 2 Theorem 1 is convenient in applications. Indeed, we may only require that the function $f$ is continuous near the zero point. In this case, there exists $M>0$ such that $f \in C[0, M]$ with $f(u)>0$ for $u \in[0, M]$. Then the function $\bar{f}$ can be defined by (4). On the other hand, the monotonicity of $f$ is not required. For example, let $f(u)=e^{u}$ and

$$
G=\left(\begin{array}{cc}
4 & -1 \\
-1 & 4
\end{array}\right)
$$

Clearly, condition (6) holds for $\varepsilon=2$. Similar arguments hold for $\gamma=1 / 4$. In view of (7), we get that $\delta=5 / 4$. Finally, by (8) we obtain

$$
\lambda_{0}=\frac{4}{25} \ln \frac{5}{4}
$$

Similar arguments hold for $f(u)=e^{-u}$ and are omitted.

The condition $f \in C[a, b]$ with $f(a) \geq a$ and $f(b) \leq b$ implies that the function $f$ has a fixed point in $[a, b]$. We now will give a more general extension.

Theorem 2 Assume that there exist $a, b>0$ with $a<b$ such that $f$ is positive and continuous on $[a, b]$,

$$
m \sum_{j=1}^{n} g_{i j}^{+}>M \sum_{j=1}^{n} g_{i j}^{-}
$$

for $i \in[1, n]$, and that $\frac{a}{A}<\frac{b}{B}$, where $m=\min _{u \in[a, b]} f(u), M=\max _{u \in[a, b]} f(u)$,

$$
A=\max _{i \in[1, n]}\left\{m \sum_{j=1}^{n} g_{i j}^{+}-M \sum_{j=1}^{n} g_{i j}^{-}\right\}, \quad \text { and } \quad B=\min _{i \in[1, n]}\left\{M \sum_{j=1}^{n} g_{i j}^{+}-m \sum_{j=1}^{n} g_{i j}^{-}\right\} \text {. }
$$

Then, for any $\lambda \in\left[\frac{a}{A}, \frac{b}{B}\right]$, problem (1) has a positive solution $x^{\lambda}$ with $x_{i}^{\lambda} \in[a, b]$ for $i \in[1, n]$. 
Proof For $x \in[a, b]^{n}$, we have

$$
\begin{aligned}
& x_{i}=\lambda \sum_{j=1}^{n} g_{i j} f\left(x_{j}\right) \\
&=\lambda\left\{\sum_{j=1}^{n} g_{i j}^{+} f\left(x_{j}\right)-\sum_{j=1}^{n} g_{i j}^{-} f\left(x_{j}\right)\right\}, \\
& \lambda\left\{m \sum_{j=1}^{n} g_{i j}^{+}-M \sum_{j=1}^{n} g_{i j}^{-}\right\} \leq x_{i} \leq \lambda\left\{M \sum_{j=1}^{n} g_{i j}^{+}-m \sum_{j=1}^{n} g_{i j}^{-}\right\}
\end{aligned}
$$

or

$$
\lambda A \leq x_{i} \leq \lambda B
$$

for $i \in[1, n]$. The proof is complete.

Remark 3 When condition (3) holds, there exists $\sigma>1$ such that

$$
\sum_{j=1}^{n} g_{i j}^{+}>\sigma \sum_{j=1}^{n} g_{i j}^{-} \quad \text { for } i \in[1, n] .
$$

In this case, we can choose suitable $a$ and $b$ such that $f$ is positive and continuous on $[a, b]$ and that $\frac{M}{m} \leq \sigma$, where $m$ and $M$ are defined in Theorem 2 .

Example 1 Let $a>4$. We consider the periodic boundary value problem

$$
\left\{\begin{array}{l}
\Delta^{2} x_{i-1}+a x_{i}=\lambda f\left(x_{i}\right) \quad \text { for } i=1 \text { or } 2, \\
x_{0}=x_{2}, \quad x_{1}=x_{3},
\end{array}\right.
$$

or the nonlinear algebraic system

$$
\left(\begin{array}{cc}
a-2 & 2 \\
2 & a-2
\end{array}\right)\left(\begin{array}{l}
x_{1} \\
x_{2}
\end{array}\right)=\lambda\left(\begin{array}{l}
f\left(x_{1}\right) \\
f\left(x_{2}\right)
\end{array}\right),
$$

or

$$
\left(\begin{array}{l}
x_{1} \\
x_{2}
\end{array}\right)=\frac{\lambda}{a(a-4)}\left(\begin{array}{cc}
a-2 & -2 \\
-2 & a-2
\end{array}\right)\left(\begin{array}{l}
f\left(x_{1}\right) \\
f\left(x_{2}\right)
\end{array}\right) .
$$

In this case, Theorems 1 and 2 are valid for (10) or (11). To the best of our knowledge, such a system cannot be handled by the previous results.

\section{Acknowledgements}

The authors thank the anonymous referees for their significant comments and suggestions to improve the quality of paper.

\section{Funding}

The third author is supported by a grant from the Natural Sciences and Engineering Research Council of Canada (NSERC), and the fourth author is supported by the Natural Science Foundation of Tianjin (No. 19JCYBJC30700). 
Availability of data and materials

Data sharing is not applicable to this paper as no datasets were generated or analyzed during the current study.

\section{Competing interests}

The authors declare that they have no competing interests.

\section{Authors' contributions}

The authors contributed equally to this paper. All authors read and approved the final manuscript.

\section{Author details}

'School of Mathematics and Statistics, Shanxi Datong University, Datong 037009, Shanxi, P.R. China. ${ }^{2}$ Department of Mathematics, Trent University, Ontario, K9L 0G2, Canada. ${ }^{3}$ School of Science, Tianjin University of Commerce, Tianjin 300134, P.R. China.

\section{Publisher's Note}

Springer Nature remains neutral with regard to jurisdictional claims in published maps and institutional affiliations.

Received: 19 May 2020 Accepted: 28 October 2020 Published online: 10 November 2020

\section{References}

1. Bendito, E., Carmona, A., Encinas, A.M.: Solving boundary value problems on networks using equilibrium measures. J. Funct. Anal. 171, 155-176 (2000)

2. Bisci, G.M., Repovš, D.: Nonlinear algebraic systems with discontinuous terms. J. Math. Anal. Appl. 398, $846-856$ (2013)

3. Bisci, G.M., Repovš, D.: Algebraic systems with Lipschitz perturbations. J. Elliptic Parabolic Equ. 1, 189-199 (2015)

4. Brouwer, L.: Über Abbildungen von Mannigfaltigkeiten. Math. Ann. 71, 97-115 (1912)

5. Candito, P., Bisci, G.M.: Existence of two solutions for a nonlinear second-order discrete boundary value problem. Adv. Nonlinear Stud. 11, 443-453 (2011)

6. Candito, P., Bisci, G.M.: Existence of solutions for a nonlinear algebraic system with a parameter. Appl. Math. Comput. 218, 11700-11707 (2012)

7. Carbonell-Nicolau, O.: On the existence of pure-strategy perfect equilibrium in discontinuous games. Games Econ. Behav. 71, 23-48 (2011)

8. Chowdhury, P.R.: Bertrand-Edgeworth equilibrium large markets with nonmanipulable residual demand. Econ. Lett. 79, 371-375 (2003)

9. Díaz, C.A., Campos, F.A., Villar, J.: Existence and uniqueness of conjectured supply function equilibria. Int. J. Electr. Power Energy Syst. 58, 266-273 (2014)

10. Du, Y.Q., Feng, W.Y., Wang, Y., Zhang, G.: Positive solutions for a nonlinear algebraic system with nonnegative coefficient matrix. Appl. Math. Lett. 64, 150-155 (2017)

11. Du, Y.Q., Zhang, G., Feng, W.Y.: Existence of positive solutions for a class of nonlinear algebraic systems. Math. Probl. Eng. 2016, Article ID 6120169 (2016)

12. Einy, E., Haimanko, O., Moreno, D., Shitovitz, B.: On the existence of Bayesian Cournot equilibrium. Games Econ. Behav. 68, 77-94 (2010)

13. Feng, W., Zhang, G.: Eigenvalue and spectral intervals for a nonlinear algebraic system. Linear Algebra Appl. 439, 1-20 (2013)

14. Goulianas, K., Margaris, A., Adamopoulos, M.: Finding all real roots of $3 \times 3$ nonlinear algebraic systems. Appl. Math. Comput. 219, 4444-4464 (2013)

15. Guo, T.G., Wang, H.Y., Wu, M.N.: Nonnegative solutions of a class of systems of algebraic equations. Dyn. Syst. Appl. 23, $211-220(2014)$

16. Li, X.J., Lu, S.P.: Periodic solutions for a kind of high-order $p$-Laplacian differential equation with sign-changing coefficient ahead of nonlinear term. Nonlinear Anal. 70, 1011-1022 (2009)

17. Lian, H., Wang, H.Y., Wu, M., Gao, T.: Positive solutions of singular algebraic systems with a parameter. Commun. Appl. Anal. 19, 565-578 (2015)

18. Liu, Y.J., Ge, W.G.: Positive solutions for $(n-1,1)$ three-point boundary value problems with coefficient that changes sign. J. Math. Anal. Appl. 282, 816-825 (2003)

19. Ma, R.Y.: Nonlinear periodic boundary value problems with sign-changing Green's function. Nonlinear Anal. 74 1714-1720 (2011)

20. Marcu, N., Bisci, G.M.: Existence and multiplicity of solutions for nonlinear discrete inclusions. Electron. J. Differ. Equ. 2012, $192(2012)$

21. Wang, H.Y., Wang, M., Wang, E.: An application of the Krasnoselskii theorem to systems of algebraic equations. J. Appl. Math. Comput. 38, 585-600 (2012)

22. Wang, Z.X., Lu, S.P., Cao, J.D.: Existence of periodic solutions for a $p$-Laplacian neutral functional differential equation with multiple variable parameters. Nonlinear Anal. 72, 734-747 (2010)

23. Wang, Z.X., Qian, L.X., Lu, S.P., Cao, J.D.: The existence and uniqueness of periodic solutions for a kind of Duffing-type equation with two deviating arguments. Nonlinear Anal. 73, 3034-3043 (2010)

24. Yang, Y., Zhang, J.: Existence results for a nonlinear system with a parameter. J. Math. Anal. Appl. 340, 658-668 (2008)

25. Yannakakis, M.: Equilibria, fixed points, and complexity classes. Comput. Sci. Rev. 3, 71-85 (2009)

26. Zeidler, E.: Nonlinear Functional Analysis and Applications I: Fixed-Point Theorems. Springer, Berlin (1986)

27. Zhang, G., Cheng, S.: Existence of solutions for solutions for a nonlinear system with a parameter. J. Math. Anal. Appl. 314, 311-319 (2006)

28. Zhang, G., Feng, W.Y.: On the number of positive solutions of a nonlinear algebraic system. Linear Algebra Appl. 422 404-421 (2007)

29. Zhang, G., Ge, S.: Existence of positive solutions for a class of discrete Dirichlet boundary value problems. Appl. Math. Lett. 48, 1-7 (2015) 